\title{
A COMPARATIVE STUDY ON ROAD TRAFFIC MANAGEMENT SYSTEMS
}

\author{
P.M. Xavier' ${ }^{1}$, Raju Nedunchezhian ${ }^{2}$ \\ ${ }^{I}$ Dean (Academic \& Student Affairs), Toc H Institute Of Science \& Technology, Kerala, India \\ ${ }^{2}$ Principal, Sri Ranganathar Institute of Engineering and Technology, TamilNadu, India
}

\begin{abstract}
With the rapid stress in infrastructure development by all nations across the world, better designed, engineered and constructed roads and highways have come up or are in different stages of implementation in rural and urban areas. With the improvement in automobile technology, lighter and high-speed vehicles are a common sight along the highways. The highway environment consists of fast and slow moving vehicles, traffic congestions particularly at traffic nodal points, collisions, accidents, bottlenecks, and all leading to unpredictability in traffic flow causing considerable loss of time, upsetting schedules, economic loss and environmental pollution. With the enhanced economic development, more and more automobiles will be on the road without commensurate improvement in road space. Having dynamic information on traffic density along highways and lateral roads will help in streamlining traffic flow. The state of road density is an important factor in evaluating travel times and travelling routes. A number of studies have been carried out on traffic management by mathematicians, engineers and town planners with varied outcomes. The studies are generally based on the volume, speed and density of vehicles on a given segment of road space using conventional traffic detection devices such as Inductive loops, magnetic loops, cameras, radars and modern Intelligent Transportation Systems (ITS) applications such as RF-Id, Wireless Sensors, VANETs and so on, each with its own limitations due to variations in prediction accuracy, environmental factors, data collection difficulties and cost. With the ubiquitous presence of mobile signals, it is now possible to monitor vehicular traffic flow along highways and lateral roads with a high degree of accuracy with minimum cost. This paper is a comparative study of existing systems for vehicular traffic management and to suggest the use of mobile signals for vehicular traffic management.
\end{abstract}

Keywords: Traffic flow, Intelligent Transportation Systems (ITS), Vehicular traffic management

\section{INTRODUCTION}

Vehicular traffic management is a major area of concern for town planners. The efforts by traffic managers and town planners to overcome traffic congestion are not achieving result as more and more vehicles are added to the road network day by day which exceeds the intended physical capacity of the road space. To tide over the traffic congestion and to ensure better traffic management, timely availability of dynamic traffic information with traffic managers are essential to regulate the traffic and divert the traffic to alternate routes.

There are several sources providing traffic information to commuters through FM stations, GPS assisted systems. However these are not covering the entire segment of population as these systems have not become mass based. Several other devices and systems such as traffic sensors, inductive loop, magnetometer, infrared devices, acoustic, ultrasonic, visual camera, radar and laser systems. As these sensors are highly sensitive to environment such as weather changes like rain, fog etc, they are less effective and error prone, costly and needs regular maintenance. None of the methods mentioned above has proved its effectiveness on a long term basis.

This paper is a comparative study and performance evaluation of various location finding techniques for mobile tracking and its application for studying traffic densities in highways and lateral roads.

\section{EXISTING METHODS}

\subsection{Global Positioning Service (GPS)}

GPS is a space-based satellite navigation system that provides location and time information in all weather conditions [1]. Any mobile station can freely use this service with a GPS receiver which calculates its position by precisely timing the signals sent by GPS. The position can be calculated if the position of each satellite and the distance from the satellite (pseudo range) is known. For accurate results four or more satellites must be visible among the 24 satellites in 4 orbital planes as shown in figure 1 .

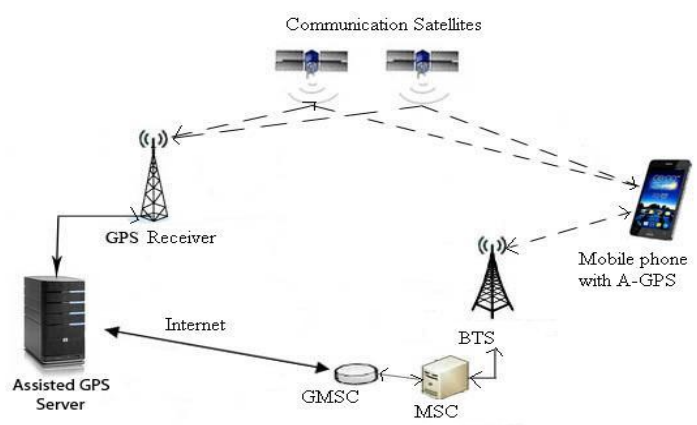

Fig- 1: Traffic detection system using GPS 
Mobile phones with GPS receiver are now entering market. However, its utilization is restricted due to high battery power consumption and need for having line of sight connectivity. Hence, its use is practically ruled out in indoor coverage and where shadow coverage is available. In addition, there is a time delay of $60-90 \mathrm{~s}$ in calculating the location process.

\subsection{Laser Tracker Technology}

Laser Technology can be used to track an object by measuring its distance [2]. The reflected laser beam is analyzed and the distance from the target is measured using either a laser interferometer or absolute distance meter (ADM).The details from the laser beam is used to find the traffic in an area using Grid based method.

This method is accurate in stable working environments. But the accuracy is reduced by atmospheric effects. Another drawback is it cannot track multiple objects in real-time as laser travels in a straight and narrow path. Also, this technique is very expensive and hence not used commonly.

\subsection{Video Based Tracking}

Use of Traffic cameras is a cost effective solution and is flexible for monitoring traffic [3]. They can be networked and remotely controlled and can cover areas of interest with its pan and zoom facility enabling coverage in a larger area. Hence, they are used for vehicle tracking particularly for vehicle counts and speed measurements. The disadvantage of this method is that to cover a longer road segment, large no. of cameras are required and hence suitable for junction monitoring.

\subsection{Infrared}

Infrared devices are useful where the visibility is poor and having limited lighting conditions [4].



Fig-2: Vehicle detection using Infrared device

Working of infrared devices for traffic detection is shown in figure 2. Based on the distance, the image quality undergoes changes and the vision getting blurred. It does pick up considerable amount of background noise. However, it is being generally used as it requires low power, and hence cost effective.

\subsection{RF-ID}

Radio Frequency Identification (RFID) is another method for vehicular tracking and traffic management [5].

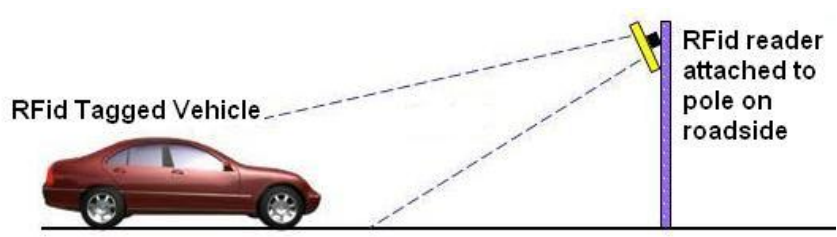

Fig-3: RFID for vehicle detection

Using this method we can uniquely identify what type of vehicle it is and we can predict the traffic accurately by analyzing the number of vehicles and their type as shown in figure 3 .

The major components of the system are:

a. the tags for identification of each vehicle

b. Interconnection with the tags

c. Processing of the information generated by the tags

\subsection{Time of Arrival (TOA)}

In this method [6] signal transmitted from the MS to the BTS is measured at the BTS. Alternatively, the signal can be initiated by the BS and the round-trip time is measured at the BTS. The distance of the MS from a BTS is related to half the round-trip time and the location of the MS is found by the intersection of three circles of appropriate radius (trilateration).

The distance is calculated by the following equation,

$$
R_{i}=C \tau_{i}=\sqrt{\left(x_{i}-X\right)^{2}+\left(y_{i}-Y\right)^{2}}
$$

Where,

C-Propagation speed of electromagnetic wave,

$\tau_{i}$-Propagation time from the mobile telephone to $\mathrm{i}^{\text {th }}$ base station,

$x_{i}, y_{i}-$ Location of $\mathrm{i}^{\text {th }}$ base station,

$\mathrm{X}$ Y-Mobile position

TOA clock inaccuracies of just $1 \mu \mathrm{s}$ will introduce a position error of $300 \mathrm{~m}$ as the velocity of the signal is $3 \times 10^{8} \mathrm{~m} / \mathrm{s}$. Hence, it requires very accurate knowledge of the start time of transmission and all receiving devices need to be synchronized.

\subsection{Angle of Arrival (AOA)}

In this method [7], Angle of Arrival (AOA) of a signal is measured. Signal from either a BTS or MS can be used for measuring the angle. The mobile is programmed to receive signals from a number of BTSs and is shown in figure 4 . In AOA method, minimum two BTSs are required for the determination of the angle. By knowing the direction of the signal with respect to the location of the BTS, it can measure the angle of incidence with respect to normal and find out the position of the MS. However, the accuracy is 
limited. AOA method is not that accurate for finding mobile location because of the following disadvantages:-

- $\quad$ Being wireless environment, obstacles, atmospheric particles, scattering etc. can cause change in angle of incidence thus leading to errors.

- AOA cannot be accurately determined if the base stations are in straight line.

- Usage of this method in indoor applications may lead to considerable inaccuracy.

AOA method requires minimum two base stations to get a location estimate. But it shows very poor performance in rural areas because of the linear orientation of base stations along major roads. Expensive antenna arrays are needed to overcome this. A small angular error of the antenna array can produce a significant error in location estimate. Also increase in the distance between the mobile stations reduces the accuracy.

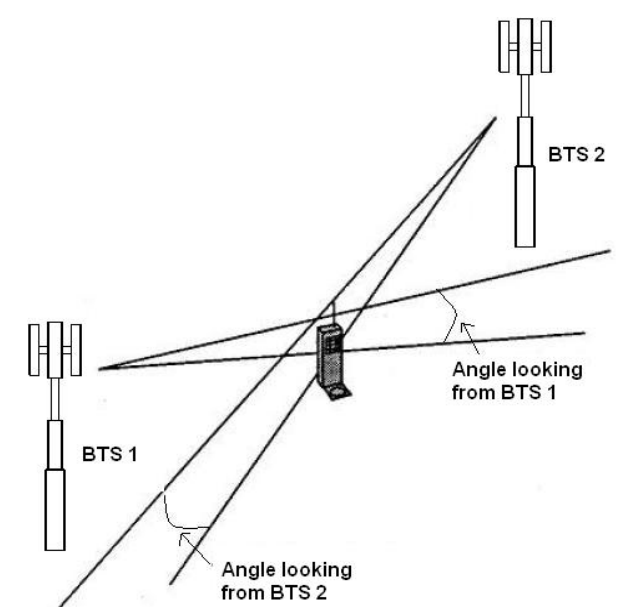

Fig- 4: Estimation of the arrival angle of the signal from a MS at a BTS

AOA method is not the first method of choices in highly dense areas where LOS is generally not available. However this technique will be used in rural areas with a reasonable amount of accuracy. The accuracy of this method is about $50 \mathrm{~m}$ in suburban environment.

\subsection{Time Difference of Arrival (TDOA)}

The BTS stations A, B and C are synchronized in order to ascertain the time difference of arrival (TDOA) of the GSM signal [8]. The time difference of arrival of the signal between station $\mathrm{A}$ and $\mathrm{B}$ is denoted $\mathrm{TDOA}_{B A}$ and describes a hyperbola curve as shown in figure 5 . When evaluating a second time difference of arrival, e.g. between station $A$ and $\mathrm{C}$ denoted $\mathrm{TDOA}_{C A}$, a second hyperbola curve leads to an intersection point which serves as the fix for the mobile phone location. For a two-dimensional localization, at least three receiving stations are necessary.

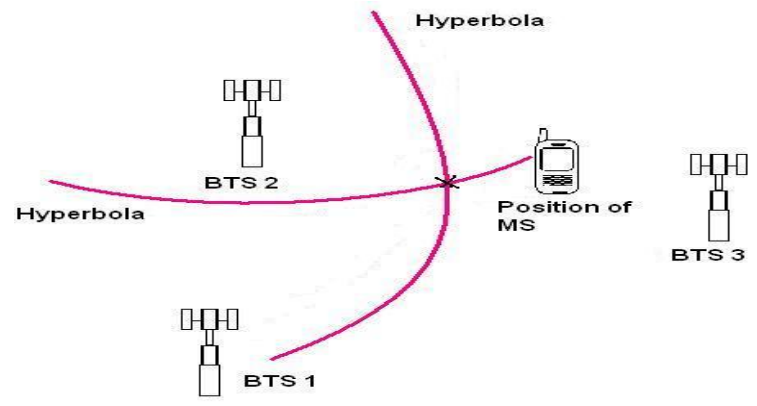

Fig-5: Time Difference of arrival

The system provides about 5-10m accuracy under favorable environmental conditions and accurate system synchronization. It is useful for locating and in search and rescue operations.

\subsection{Observed Time Difference of Arrival (OTD)}

This mechanism has each MS monitor the time differences between the epochs of the different BSs in its vicinity [9]. These observed time differences are obtainable in both the idle and communication modes of a MS and are used to estimate the change in timing advance or retard required at handover to another BS. Positional location can be derived by comparing OTDs from a number of BSs.

But it is difficult to implement in rural and hill stations as BSS is very small.

\subsection{Enhanced Observed Time Difference of} Arrival (E-OTD)

The E-OTD operates only on GSM network [10]. A location estimate can be produced in both idle and call modes. The E-OTD method is based on measurements in the MS of the relative time of arrival bursts received from serving BTS and at least one pairs of BTSs.

Figure 6 explains the functioning of the E-OTD method. The measurement units are dispersed in a larger area. The base station broadcasts a signal every 30 second in an asynchronous manner. Based on the signal from a minimum of three base stations by the handset and LMU, the time difference in arrival of the signal from each BS at the handset and LMU are calculated. The differences in times are combined and the location of the mobile station is found out by intersection. It is one of the accurate methods which have an accuracy of 60 to 125 meters.

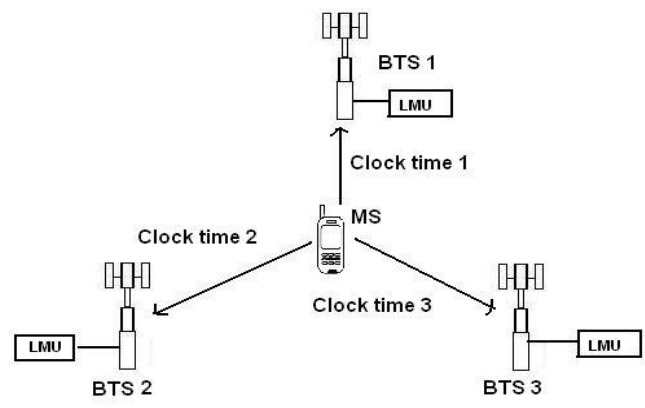

Fig-6: E-OTD 
The disadvantages of this method include modification of handset with E-OTD software, installation of LMU for infrastructure and multipath problems.

\section{CONCLUSION}

Location based services (LBS) as seen from the above discussions is useful in tracking and locating. However, the mobile number of the individual is to be known. The accuracy of the systems depends on timing synchronization, availability of line-of-sight, conducive environmental conditions, orientation of the BTS sites and so on. LBS in general is not a solution for use as a traffic management tool. Systems such as Video based tracking, Infrared and RF-Id, are useful in monitoring and counting of traffic flow. However, these devices also have limitations due to environmental, weather, ambient temperature differences, cost and frequent maintenance requirements. Out of all devices discussed, GPS is a better method with better accuracy but is costly and is not likely to be universally equipped in the near future. Therefore the existing location based systems are not sufficient for mobile tracking and we need to find alternatives.

Since nineties, considerable amount of research is being done in utilizing mobile signals for vehicular traffic management. The advantage of using mobile network for traffic management is that it is cost effective as the infrastructure already exists and the mobile signals are ubiquitous. The location of the MS if tracked continuously can predict the movement of vehicles as it is presumed that each and every vehicle will have a minimum of one mobile phone.

\section{REFERENCES}

[1]. Jie Du , Barth M.J. ,"Next-Generation Automated Vehicle Location Systems: Positioning at the Lane Level ", Intelligent Transportation Systems, IEEE Transactions, March 2008.

[2]. Huijing Zhao Key, Chiba Masaki; Shibasaki R.; Shao Xiaowei; Jinshi Cui;Hongbin Zha , "A Laser-ScannerBased Approach Toward Driving Safety and Traffic Data Collection", Intelligent Transportation Systems, IEEE Transactions, Sept. 2009.

[3]. Kumar P, SurendraRanganath, HuangWeimin, Sengupta K, "Framework for real-time behavior interpretation from traffic video", Intelligent Transportation Systems, IEEE Transactions, March2005. [4]. Nelson B.N, "Automatic vehicle detection in infrared imagery using a fuzzy inference-based classification system" Fuzzy Systems, IEEE Transactions, Feb 2001.

[5]. Hongzi Zhu, Shanghai Minglu Li, Yanmin Zhu, Ni L.M, "HERO: Online Real-Time Vehicle Tracking", Parallel and Distributed Systems, IEEE Transactions, May 2009.

[6]. K. W. Cheung, H. C. So, W.K. Ma, and Y. T. Chan, "Least Squares Algorithms for Time-of-Arrival-Based Mobile Location", IEEE Transactions on Signal Processing, VOL. 52, NO. 4, APRIL 2004.

[7]. Xin Wang, Zongxin Wang, and Bob O'Dea, "A TOABased Location Algorithm Reducing the Errors Due to Non-

Line-of-Sight (NLOS)" Vehicular Technology, VOL. 52, NO. 1, January 2003.

[8]. Alexander Goetz, Stefan Zorn, Richard Rose, Georg Fischer and Robert Weigel "A Time Difference of Arrival System Architecture for GSM Mobile Phone Localization in Search and Rescue Scenarios", 8th Workshop on Positioning Navigation and Communication (WPNC), 2011, DOI: 10.1109/WPNC.2011.5961009, Page(s): 24- 27.

[9]. Singh K, Ismail M, "OTDOA location determining technology for universal intelligent positioning system(UIPS)implementation in Malaysia", Networks, 2005. Jointly held with the 2005 IEEE 7th Malaysia International Conference on Communication. 2005 13th IEEE International Conference on 16-18 Nov. 2005

[10]. S. Larder, "E-OTD-based location service implementation", Mobile Location Workshop (MLW 2001), Espoo, Finland, June 2001.

[11]. KaiYang, Jianping An, Xiangyuan Bu, Gangcan Sun, "Constrained Total Least-Squares Location Algorithm Using Time-Difference-of-Arrival Measurements", Vehicular Technology, IEEE Transactions on March 2010.

[12]. Haibin Ling1, Li Bai, Erik Blasch, and Xue Mei, "Robust Infrared Vehicle Tracking across Target Pose Change using L1 Regularization", Information Fusion (FUSION), 2010 13th Conference, July 2010

[13]. Alessandro Andreadis, Giuliano Benelli, Alberto Bianchi, Pasquale edele, Giovanni Giambene, "Universal Access to Personalised Information Services", IST Mobile Summit, Barcelona, October 2001

[14]. Isaac K Adusei, K. Kyamakya, Klaus Jobmann, "Mobile Positioning Technologies in Cellular Networks: A valuation of their Performance Metrics", 0-7803-76250/02/2002 IEEE.

[15]. Heikki Laitinen, Suvi Ahonen, Sofoklis Kyriazakos, Jaakko Lähteenmäki, Raffaele Menolascino, Seppo Parkkila, "Cellular network optimisation based on mobile Location”, CELLO-WP2-VTT-D03-007-Int, 5 Nov 2001.

[16]. Mika Keski-Heikkilä , "End-user Location in Digital Mobile Networks", S-72.333, 19 Feb 2002.

[17]. S.C. Swales, J.E. Maloney, and J.O. Stevenson, "Locating mobile phones \& the US wireless E-911 mandate", IEE colloquium on novel methods of location and tracking of cellular mobiles and their system applications, London, May 1999

[18]. Robin Wentao Ouyang, Albert Kai-Sun Wong, "An Enhanced TOA-based Wireless Location Estimation Algorithm for Dense NLOS Environments", IEEE Wireless Communications and Networking Conference, 2009.

\section{BIOGRAPHIES}

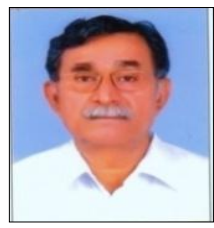

communication,
Prof. (Col.) P. M. Xavier holds B. Tech degree in Telecommunication from JNU, Delhi, in 1979 and M. Tech Degree in Electronics from Central University Allahabad, Uttar Pradesh, in 1990. Major fields of interests includes microwave mobile cellular systems and optoelectronics. He joined the Indian Army in the year 1967 and retired as Colonel from Corps of Signals. On retirement, he served with Birla AT\&T as GM Engineering/Project till 
2001. Thereafter, he worked with Alcatel-Lucent till 2007 as Regional Manager. He has been with Toc $\mathrm{H}$ institute of Science \& Technology as Professor since 2007, teaching and guiding both B. Tech \& M. Tech students and presently he is Dean (Academics \& Student Affairs) of the institute.

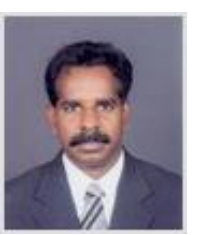

Prof. (Dr.) Raju Nedunchezhian is the Principal of Sri Ranganathar Institute of Engineering \& Technology, Coimbatore, Tamilnadu. Prior to this, he was VicePrincipal of Kalaignar Karunanidhi Institute of Technology, Coimbatore, TamilNadu. He also worked as Research Coordinator of the Institute and Head of Computer Science and Engineering Department (PG) at Sri Ramakrishna Engineering College, Coimbatore. He has more than 22 years of experience in research and teaching. He obtained his BE (Computer Science and Engineering) degree in 1991, ME (Computer Science and Engineering) degree in 1997 and Ph.D (Computer Science and Engineering) in 2007. He has guided many UG, PG, M.Phil and $\mathrm{Ph}$. D scholars. Currently, he is research guide for many $\mathrm{Ph}$. D scholars of the Anna University, Coimbatore, and Bharathiar University. 